CARNETS DE Carnets de géographes

GÉOGRAPHES

4 | 2012

Géographies critiques

\title{
Herodote (1977-4 et 1978-1), « l'enquête et le terrain »
}

Yann Calberac

\section{(2) OpenEdition}

Journals

Édition électronique

URL : http://journals.openedition.org/cdg/1013

DOI : $10.4000 /$ cdg. 1013

ISSN : 2107-7266

Éditeur

UMR 245 - CESSMA

Référence électronique

Yann Calberac, « Herodote (1977-4 et 1978-1), «l'enquête et le terrain » », Carnets de géographes [En ligne], 4 | 2012, mis en ligne le 01 septembre 2012, consulté le 24 septembre 2020. URL : http:// journals.openedition.org/cdg/1013; DOI : https://doi.org/10.4000/cdg.1013

\section{(c) $\oplus \Theta \Theta$}

La revue Carnets de géographes est mise à disposition selon les termes de la Licence Creative Commons Attribution - Pas d'Utilisation Commerciale - Pas de Modification 4.0 International. 


\title{
HERODOTE (1977-4 ET 1978-1), « L'ENQUÊTE ET LE TERRAIN »
}

\author{
Compte rendu de lecture par YANN CALBERAC \\ ATER en géographie \\ Université Paris-Sorbonne (IUFM de Paris) \\ UMR 8185 ENeC \\ yann.calberac@ens-lyon.org
}

Ces deux volumes sont disponibles en ligne dans Gallica :

- « L'enquête et le terrain (1977-4) : http://gallica.bnf.fr/ark:/12148/bpt6k5622671r.image.swf

- « L'enquête et le terrain (2)» (1978-1) : http://gallica.bnf.fr/ark:/12148/bpt6k5797635m.image.swf

Les pensées critiques émergent de l'Université française dans les années 1960 et 1970, au moment même où la géographie, qui est alors un peu marginale dans le paysage académique en dépit du rayonnement qui a été le sien dans la première moitié du $X^{\mathrm{e}}$ siècle, connaît une crise identitaire sans précédent. Cette dernière est d'abord scientifique: le paradigme classique est contesté de toute part, et la géographie d'inspiration vidalienne perd son souffle (Orain, 2009). Elle est aussi générationnelle: alors que la thèse d'Etat représentait toujours le sésame de la carrière universitaire, la massification de l'enseignement supérieur entamée dès le début des années 1960 permet l'entrée soudaine de jeunes assistants, ce qui a pour but de redéfinir les positions au sein du champ académique (Bourdieu, 1984). Enfin, cette période constitue aussi la renégociation de l'économie générale des discours disciplinaires (Calbérac, 2010). La géographie se pose donc la question de sa pertinence scientifique et de son utilité sociale (Calbérac et Delage, 2010).

De fait, si les géographes se frottent à la critique, c'est d'abord contre leur discipline qu'il convient de rénover, comme le rappelle Bernard Bret dans l'entretien publié dans ce numéro ou Jacques Lévy dans l'entretien publié dans le premier numéro des Carnets. En effet, la géographie - que Vidal de La Blache a définie comme l'étude des relations homme / milieu à l'échelle régionale - n'est plus opératoire pour comprendre les évolutions que connaissent les sociétés à toutes les échelles, comme la bipolarisation et la montée du Tiers-monde (selon l'expression de l'époque), l'explosion démographique et l'urbanisation, ou, pour le cas français, les mutations socio-économiques structurelles des Trente Glorieuses. La géographie est d'autant moins opératoire pour étudier ces dynamiques qu'elle repose sur un équilibre instable: Emmanuel de Martonne a 
volontiers tiré la géographie vers son seul volet physique et la place des hommes en société n'est pas, à l'époque, le cœur de la discipline. L'enjeu de ces débats est donc de refonder l'objet de cette discipline et de la repositionner dans le champ scientifique.

A cette critique scientifique s'ajoute également une dimension politique : les géographes de l'époque réévaluent l'héritage idéologique de la géographie vidalienne et interrogent le contexte politique qui a permis son essor et son institutionnalisation. En effet, pour lui faire une place dans l'Université républicaine qui se met alors en place, Vidal donne à la géographie l'apparence de la neutralité axiologique, alors qu'elle répond - de manière implicite mais incontestable - au programme idéologique de la Troisième République (Legris, 2005). La géographie alimente donc le discours républicain sur la nation et son territoire et joue un rôle actif dès lors qu'il s'agit d'unifier le territoire, de coloniser ou de préparer la revanche contre l'Allemagne.

C'est dans ce contexte qu'émergent des propositions qui visent toutes à refonder la discipline, et à redéfinir ses méthodes et ses objets. Celle d'Yves Lacoste se fait alors particulièrement entendre. Il travaille sur le Tiers-Monde et le sous-développement (par exemple Lacoste, 1965) et participe, depuis 1969, à l'aventure du Centre Universitaire de Vincennes qui donnera naissance à l'Université de Saint-Denis. Fort de ses engagements communistes, tiers-mondistes et anticolonialistes, il cherche à refonder le contrat politique de la géographie en rompant le lien qui unissait la géographie vidalienne au pouvoir politique de la Troisième République et en faisant de la géographie une arme qui serait utilisable dans toutes les luttes politiques ou économiques. Il publie à cet effet en 1976 un pamphlet dont le titre est resté célèbre: La géographie, ça sert, d'abord, à faire la guerre. Yves Lacoste part d'un constat : seuls les militaires ont compris l'intérêt de la géographie, eux qui l'utilisent comme l'arme qu'elle a toujours été et qui la mobilisent à des fins stratégiques ou tactiques dans la conduite des opérations militaires. Rien à voir avec la "géographie des maîtres », c'est-à-dire la géographie vidalienne, qui constitue une " discipline bonasse » dénuée de tout intérêt. Yves Lacoste appelle donc à retourner la géographie contre ceux qui l'utilisent à des fins de domination, à dénoncer ces formes de domination et à lutter contre elles : il invite à une guérilla dont l'arme principale serait la géographie, et propose à cet effet de réinvestir le champ de la géopolitique qui, depuis la seconde guerre mondiale, était tombée dans l'ère du soupçon. La géographie se mue donc en une géopolitique définie comme l'analyse des différentes formes de rivalités de pouvoir sur un territoire à toutes les échelles. Pour mettre en œuvre ce projet scientifique et politique, Yves Lacoste s'entoure d'une équipe et crée la revue Hérodote publiée par l'éditeur engagé François Maspero.

Deux numéros parmi les premiers - intitulés «L'enquête et le terrain » - sont consacrés à la question du terrain. Ils prolongent la réflexion de La géographie, ça sert, d'abord, à faire la guerre en explorant cette fois la dimension méthodologique de la géographie et les nouvelles méthodes à mettre en œuvre pour rénover la pratique disciplinaire. Ces deux numéros constituent également, en plein cœur de la crise de la géographie, la première arène réflexive et collective sur le terrain, envisagé comme la pratique intrinsèque du métier de géographe et qui consiste en la collecte in situ des données, au contact des populations étudiées. 
L'occasion de cette réflexion est donnée par la réception du numéro inaugural d'Hérodote paru en 1976. La vieille garde des géographes n'a pas pardonné à Yves Lacoste d'avoir publié, après son enquête sur les bombardements américains au Vietnam, des extraits de la conclusion de la thèse de Pierre Gourou : dans ces pages rassemblées sous le titre «Les beautés du delta » on trouve une description paysagère qui mobilise un imaginaire exotique qui n'aborde pas le problème politique de la colonisation. Yves Lacoste est donc accusé d'avoir voulu ridiculiser Pierre Gourou qui est alors 1'une des figures majeures de la géographie française. Dans l'éditorial du numéro 8 dont on rend compte ici, Yves Lacoste se défend d'avoir voulu attaquer Pierre Gourou : ce n'est pas tant à lui qu'à la géographie vidalienne qui a fait du paysage son objet privilégié qu'il s'en prend, et prône une approche qui permettrait au géographe de comprendre les enjeux de la situation dans laquelle il mène son enquête. Yves Lacoste enfonce le clou: c'est la lecture attentive de la thèse de Pierre Gourou qui lui a apporté les éléments d'hydrologie nécessaires pour comprendre l'impact qu'auraient les bombardements sur les digues du fleuve rouge. Preuve que la géographie peut apporter un savoir opératoire pour peu qu'on le mobilise comme il convient. Le ton est donné : c'est - dans la foulée de La géographie, ça sert, d'abord, à faire la guerre - de l'engagement du chercheur dans la situation qu'il étudie qu'il faut discuter. En dissimulant les réalités sociales derrière l'écran de fumée des «beautés du delta », la géographie participe autant d'une mythification que d'une mystification menée aux profits des puissants, en l'occurrence les états-majors, et au détriment des populations concernées en premier lieu, à savoir les habitants. La preuve : dès que la teneur réelle du projet américain a été éventée par les prises de position médiatiques de Yves Lacoste, les bombardements ont aussitôt cessé (Lacoste, 2010). Le collectif réuni au sein d'Hérodote entend donc refonder la relation d'enquête : les géographes ne doivent plus dominer en les regardant de haut (comme les y invite l'analyse paysagère vidalienne) les sociétés qu'ils étudient, mais ils sont désormais invités à faire une géographie à côté des populations qu'ils viennent enquêter et qui ne pourra pas leur nuire.

Le cœur du dispositif est occupé par un texte mis en discussion dans le numéro 8 que les articles des deux numéros viennent éclairer et enrichir, ce qui produit un dialogue fécond. C'est l'ethnologue Camille Lacoste-Dujardin, spécialiste des Berbères et de la Kabylie, qui rédige ce texte liminaire dans lequel elle interroge la posture des chercheurs sur le terrain : comment peuvent-ils aujourd'hui être à côté des populations pour leur soutirer des informations sans lesquelles leurs recherches (et donc l'avancée de leur carrière) seraient impossibles, et être demain aux côtés des puissants qui vont mobiliser leur savoir au détriment des populations enquêtées ? Camille Lacoste-Dujardin insiste donc non seulement sur l'engagement du chercheur qui ne doit plus être l'allié des puissants mais aussi sur la nécessaire restitution des savoirs aux populations enquêtées. A la question éthique (le don et le contre-don) se rajoute une question stratégique : permettre aux populations de lutter à armes égales avec les puissants.

Ce débat est novateur sur le fond - (re)politiser la géographie - mais aussi dans la forme, originale, de la discussion que l'on retrouve dans une autre revue, fondée un an avant Hérodote, L'espace géographique: il s'agit alors de rompre avec les formes héritées du 
débat disciplinaire tel qu'il était porté par les anciennes revues, notamment les Annales de géographie. Bien plus, ces deux numéros ouvrent la géographie à d'autres disciplines (comme l'histoire avec François Hartog qui, dans le numéro 9, interroge la figure historique d'Hérodote, le premier géographe, et étudie la méthode qu'il met en œuvre dans ses Enquêtes) et il n'est guère étonnant que ces interrogations soient portées principalement par des ethnologues, notamment Claude Lévi-Strauss qui participe à ce numéro, dans la mesure où ils se sont précocement interrogés sur leur engagement sur le terrain.

Cette volonté de faire entendre des points de vue extérieurs à la discipline se double du désir de faire entendre ceux, dans la discipline, que l'on n'a pas l'habitude d'entendre dans les revues de l'époque : les étudiants. Ces numéros sont ainsi l'occasion de réfléchir au travail des jeunes chercheurs ainsi qu'à la dimension pédagogique de la discipline. De même que l'on invite à abandonner l'approche paysagère de la géographie vidalienne, de même faut-il abandonner les formes de l'excursion pédagogique qui consiste bien souvent à un cours magistral en extérieur et à un commentaire de paysages. Hérodote privilégie la forme du stage qui plonge quelques jours durant les étudiants dans une société et un territoire dont ils doivent démêler les enjeux et les tensions. Un tel stage est même raconté du point de vue d'un habitant de la commune varoise de Fayence qui a vu débarquer en juillet 1976 des étudiants de l'Université de Vincennes qui ont enquêté et qui ont proposé le dernier jour de leur séjour une exposition sur le marché pour restituer le fruit de leur travail.

Ces deux numéros s'inscrivent donc dans une démarche doublement critique : d'une part à l'égard d'une discipline dont il s'agit de renégocier le contrat politique (passer d'une discipline qui a été l'un des vecteurs idéologiques de la Troisième République à une discipline engagée dans les luttes politiques et anticoloniales), et d'autre part à définir une éthique du chercheur engagé. Un paradoxe demeure: alors que Yves Lacoste s'en prend avec véhémence à la géographie vidalienne, il conforte pourtant, pour refonder la discipline, une pratique instituée et institutionnalisée en son temps par Vidal de La Blache : le terrain. Le renouvellement de la géographie ainsi proposé ne passe pas par une remise en cause du terrain. Bien au contraire: dans un article proposé dans le numéro 9, Bernard Kayser fait de l'enquête de terrain la seule légitimité scientifique dont peuvent s'enorgueillir les géographes, et reprend à son compte la formule de Mao que le Petit livre rouge a rendu populaire : «Sans enquête, pas de droit à la parole ! »

\section{Bibliographie}

Bourdieu P. (1984) Homo academicus, Paris, Les éditions de Minuit, 302 p.

Calbérac Y. (2010) Terrains de géographes, géographes de terrain. Communauté et imaginaire disciplinaires au miroir des pratiques de terrain des géographes français du $\mathrm{XX}^{\mathrm{e}}$ siècle. Thèse de doctorat en Géographie, Université Lumière Lyon 2. URL: http://tel.archives-ouvertes.fr/tel-00551481 
Calbérac Y. et Delage A. (2010) «Introduction. L'approche spatiale comme moyen de compréhension et d'action sur les sociétés ». Dossier «A quoi sert la géographie?» Tracés, revue de sciences humaines, hors-série, pp. 121-134.

Lacoste Y. (1965) Géographie du sous-développement, Paris, PUF, 284 p.

Lacoste Y. (1976) La géographie, ça sert, d'abord, à faire la guerre, Paris, Maspero, $187 \mathrm{p}$.

Lacoste Y. (2010) La géopolitique et le géographe. Entretiens avec Pascal Lorot, Paris, Choiseul, $268 \mathrm{p}$.

Legris P. (2005) Vidal de La Blache : un savant face à la Troisième République, DEA de Sciences politiques, Université Paris 1 Panthéon-Sorbonne. URL : http://www.univparis1.fr/IMG/pdf/legris-DEA.pdf

Orain, O. (2009) De plain-pied dans le monde, écriture et réalisme dans la géographie française au $\mathrm{XX}^{\circ}$ siècle, Paris, L'Harmattan, 427 p. 